UDC 524.834

PACS 98.80.Cq,

DOI: $10.22363 / 2658-4670-2020-28-2-131-140$

\title{
Spinor field in a spherically symmetric Friedmann Universe
}

\author{
Saha Bijan ${ }^{1,2}$, Evgeniy I. Zakharov ${ }^{1}$, Victor S. Rikhvitsky ${ }^{2}$ \\ ${ }^{1}$ Institute of Physical Research and Technology \\ Peoples' Friendship University of Russia (RUDN University) \\ 6, Miklukho-Maklaya St., Moscow, 117198, Russian Federation \\ ${ }^{2}$ Laboratory of Information Technologies \\ Joint Institute for Nuclear Research \\ 6, Joliot-Curie St., Dubna, Moscow region, 141980, Russian Federation
}

(received: April 18, 2020; accepted: June 30, 2020)

In recent years spinor field is being used by many authors to address some burning issues of modern cosmology. The motive behind using the spinor field as a source for gravitational field lies on the fact that the spinor field not only can describe the different era of the evolution but also can simulate different substances such as perfect fluid and dark energy. Moreover, the spinor field is very sensitive to the gravitational one and depending on the gravitational field the spinor field can react differently and change the spacetime geometry and the spinor field itself differently. This paper provides a brief description of the nonlinear spinor field in the FriedmannLemaitre-Robertson-Walker (FLRW) model. The results are compared in Cartesian and spherical coordinates. It is shown that during the transition from Cartesian coordinates to spherical ones, the energy-momentum tensor acquires additional nonzero non-diagonal components that can impose restrictions on either spinor functions or metric ones.

Key words and phrases: spinor field, FLRW model, Cartesian coordinates, spherical coordinates

\section{Introduction}

In 1998, it was found that the universe is not just expanding, but doing so with acceleration. Many hypotheses are proposed to explain this phenomenon. The most significant of them is the hypothesis of the existence of dark energy, which evenly fills the entire Universe and has a negative pressure. Some perfect liquid or scalar field is used to describe dark energy.

But there is another approach. Using the spinor field as a source of gravity. In recent years it was shown that the spinor field can give rise to a singularity-free Universe [1]-[5]. Beside this the spinor field can accelerate

(C) Bijan S., Zakharov E.I., Rikhvitsky V.S., 2020

This work is licensed under a Creative Commons Attribution 4.0 International License

http://creativecommons.org/licenses/by/4.0/ 
the isotropization process of the initially anisotropic spacetime [3], [4], [6], [7]. Finally, the spinor field can be considered as an alternative model for dark energy [7]-[17].

Moreover, it was shown that spinor field is very sensitive to gravitational one [18] and its specific behavior in presence of the gravitational field can alter the geometry of the spacetime as well as the components of the spinor field itself [19].

This is possible due to the specific behavior of the spinor field in the presence of a gravitational field. The spinor field in cosmological models has already been considered in [3], [9], [20]. But in all these works, the spinor field is considered in Cartesian coordinates. A spinor field in spherically symmetric spaces was considered in [21]-[23].

As can be seen from all these works, non-diagonal components of the energy-momentum tensor can impose additional restrictions on either metric functions or spinor functions. In this paper we consider the spinor field in the framework of a spherically symmetric FLRW model. The results are compared with those obtained in Cartesian coordinates.

\section{Basic equations}

The action for a gravitational field and a nonlinear spinor field can be written as follows:

$$
S(g, \psi, \bar{\psi})=\int\left(\frac{R}{2 \varkappa}+L_{s p}\right) d \Omega
$$

where $R$ is a Ricci scalar, $\varkappa=8 \pi G, G$ is a gravitational constant, $L_{s p}$ is a Lagrangian for a nonlinear spinor field, which looks like this:

$$
L_{s p}=\frac{i}{2}\left(\bar{\psi} \gamma^{\mu} \nabla_{\mu} \psi-\nabla_{\mu} \bar{\psi} \gamma^{\mu} \psi\right)-m \bar{\psi} \psi-F
$$

where $m$ is the mass, $F=F(K)$ is the nonlinear term. The $K$ parameter takes one of 4 values: $I, J, I+J, I-J$. Here $I=S^{2}=(\bar{\psi} \psi)^{2}$ and $J=P^{2}=\left(\bar{\psi} \gamma^{5} \psi\right)^{2}$.

From the expression (2), we can get the equations for the spinor field:

$$
\begin{aligned}
& i \gamma^{\mu} \nabla_{\mu} \psi-m \psi-D \psi-i \Upsilon \gamma^{5} \psi=0 \\
& i \nabla_{\mu} \bar{\psi} \gamma^{\mu}+m \bar{\psi}+D \bar{\psi}+i \Upsilon \bar{\psi} \gamma^{5}=0
\end{aligned}
$$

where the following symbols are entered:

$$
\begin{aligned}
& D=2 S F_{K} K_{I}=2 S \frac{d F}{d K} \frac{d K}{d I}, \\
& \Upsilon=2 P F_{K} K_{J}=2 P \frac{d F}{d K} \frac{d K}{d J} .
\end{aligned}
$$


From (2), (3) and (4) an alternative form of the Lagrangian can be obtained:

$$
L=2 K \frac{d F}{d K}-F(K)
$$

The covariant derivatives $\nabla_{\mu}$ are defined as follows:

$$
\begin{aligned}
& \nabla_{\mu} \psi=\partial_{\mu} \psi-\Gamma_{\mu} \psi, \\
& \nabla_{\mu} \bar{\psi}=\partial_{\mu} \bar{\psi}+\bar{\psi} \Gamma_{\mu},
\end{aligned}
$$

where $\Gamma_{\mu}$ is a spinor affine connection that is defined as follows:

$$
\Gamma_{\mu}=\frac{1}{4} g_{\rho \delta}\left(\frac{\partial e_{\sigma}^{(b)}}{\partial x^{\mu}} e_{(b)}^{\rho}-\Gamma_{\mu \sigma}^{\rho}\right) \gamma^{\delta} \gamma^{\sigma},
$$

where $e_{\sigma}^{(b)}$ is a system of orthogonal 4-vectors that obey the following expressions:

$$
\begin{gathered}
e_{\mu}^{(a)} e_{(a)}^{\nu}=\delta_{\mu}^{\nu}, \quad e_{\mu}^{(a)} e_{(b)}^{\mu}=\delta_{b}^{a}, \\
g_{\mu \nu}(x)=e_{\mu}^{a}(x) e_{\nu}^{b}(x) \eta_{a b} .
\end{gathered}
$$

The expression for the energy-momentum tensor is as follows:

$$
T_{\mu}^{\rho}=g^{\rho \nu} \bar{T}_{\nu \mu}-g^{\rho \nu} \tilde{T}_{\nu \mu}-\delta_{\mu}^{\rho}\left[2 K F_{K}-F(K)\right],
$$

where the following symbols are used:

$$
\begin{gathered}
\bar{T}_{\nu \mu}=\frac{i}{4}\left(\bar{\psi} \gamma_{\mu} \partial_{\nu} \psi+\bar{\psi} \gamma_{\nu} \partial_{\mu} \psi-\partial_{\mu} \bar{\psi} \gamma_{\nu} \psi-\partial_{\nu} \bar{\psi} \gamma_{\mu} \psi\right), \\
\tilde{T}_{\nu \mu}=\frac{i}{4} \bar{\psi}\left(\gamma_{\mu} \Gamma_{\nu}+\Gamma_{\nu} \gamma_{\mu}+\gamma_{\nu} \Gamma_{\mu}+\Gamma_{\mu} \gamma_{\nu}\right) \psi
\end{gathered}
$$

\section{Cartesian coordinates}

This section uses the following metric:

$$
d s^{2}=d t^{2}-a^{2}(t)\left[d x^{2}+d y^{2}+d z^{2}\right] .
$$

Nontrivial components of the Einstein tensor in this metric have the following form:

$$
\begin{gathered}
G_{0}^{0}=-3 \frac{\dot{a}^{2}}{a^{2}}, \\
G_{j}^{i}=-\left(2 \frac{\ddot{a}}{a}+\frac{\dot{a}^{2}}{a^{2}}\right) \quad i, j=1,2,3 .
\end{gathered}
$$


From metric (14), we can use (10) to find expressions for tetrads:

$$
e_{0}^{(0)}=1 \quad e_{1}^{(1)}=a(t) \quad e_{2}^{(2)}=a(t) \quad e_{3}^{(3)}=a(t) .
$$

Expressions for $\Gamma_{\mu}$ are obtained from (17) and (8):

$$
\Gamma_{0}=0 \quad \Gamma_{1}=\frac{\dot{a}}{2} \bar{\gamma}^{1} \bar{\gamma}^{0} \quad \Gamma_{2}=\frac{\dot{a}}{2} \bar{\gamma}^{2} \bar{\gamma}^{0} \quad \Gamma_{3}=\frac{\dot{a}}{2} \bar{\gamma}^{3} \bar{\gamma}^{0} .
$$

From (3), (4) (6), (7) and (18) we get the equations for the spinor field:

$$
\begin{aligned}
& i \bar{\gamma}^{0}\left(\dot{\psi}+\frac{3}{2} \frac{\dot{a}}{a} \psi\right)-m \psi-D \psi-i \Upsilon \gamma^{5} \psi=0, \\
& i\left(\dot{\bar{\psi}}+\frac{3}{2} \frac{\dot{a}}{a} \bar{\psi}\right) \bar{\gamma}^{0}+m \bar{\psi}+D \bar{\psi}+i \Upsilon \bar{\psi} \gamma^{5}=0 .
\end{aligned}
$$

Non-trivial components of the energy-momentum tensor are obtained from (11), (12), (13), (18), (19) and (20):

$$
\begin{gathered}
T_{0}^{0}=m S+F(K), \\
T_{1}^{1}=F(K)-2 K F_{K}, \\
T_{2}^{2}=F(K)-2 K F_{K}, \\
T_{3}^{3}=F(K)-2 K F_{K} .
\end{gathered}
$$

The complete system of Einstein equations looks like this:

$$
\begin{gathered}
3 \frac{\dot{a}^{2}}{a^{2}}=8 \pi G(m S+F(K)), \\
2 \frac{\ddot{a}}{a}+\frac{\dot{a}^{2}}{a^{2}}=8 \pi G\left(F(K)-2 K F_{K}\right) .
\end{gathered}
$$

A more detailed description of this case can be found in the work [24].

\section{Spherical coordinates}

A completely different situation occurs when moving from Cartesian coordinates to spherical ones. The transition is performed as follows:

$$
\begin{gathered}
x=r \sin (\theta) \cos (\varphi), \\
y=r \sin (\theta) \sin (\varphi), \\
z=r \cos (\theta) .
\end{gathered}
$$

The following metric is obtained from (27), (28), (29) and (14):

$$
d s^{2}=d t^{2}-a^{2}(t)\left[d r^{2}+r^{2}\left(d \theta^{2}+\sin ^{2}(\theta) d \varphi^{2}\right)\right] .
$$


The nontrivial components of the Einstein tensor remain unchanged. Expressions for tetrads are obtained from (30) using (10):

$$
e_{0}^{(0)}=1 \quad e_{1}^{(1)}=a(t) \quad e_{2}^{(2)}=a(t) r \quad e_{3}^{(3)}=a(t) r \sin (\theta) .
$$

Expressions for affine connectivity are obtained from (31) and (8):

$$
\begin{gathered}
\Gamma_{0}=0 \\
\Gamma_{1}=\frac{1}{2} \dot{a} \bar{\gamma}^{1} \bar{\gamma}^{0}, \\
\Gamma_{2}=\frac{1}{2}\left(\dot{a} r \bar{\gamma}^{2} \bar{\gamma}^{0}+\bar{\gamma}^{2} \bar{\gamma}^{1}\right), \\
\Gamma_{3}=\frac{1}{2}\left(\dot{a} r \sin (\theta) \bar{\gamma}^{3} \bar{\gamma}^{0}+\sin (\theta) \bar{\gamma}^{3} \bar{\gamma}^{1}+\cos (\theta) \bar{\gamma}^{3} \bar{\gamma}^{2}\right) .
\end{gathered}
$$

Substituting (32), (33), (34) and (35) into (3) and (4) is obtained:

$$
\begin{aligned}
& i \bar{\gamma}^{0} \dot{\psi}+i \frac{3}{2} \frac{\dot{a}}{a} \bar{\gamma}^{0} \psi+i U \bar{\gamma}^{1} \psi+i V \bar{\gamma}^{2} \psi-[m+D] \psi-i \Upsilon \gamma^{5} \psi=0, \\
& i \dot{\bar{\psi}} \bar{\gamma}^{0}+i \frac{3}{2} \frac{\dot{a}}{a} \bar{\psi} \bar{\gamma}^{0}+i U \bar{\psi} \bar{\gamma}^{1}+i V \bar{\psi} \bar{\gamma}^{2}+[m+D] \bar{\psi}+i \Upsilon \bar{\psi} \gamma^{5}=0
\end{aligned}
$$

where $U=1 / a r$ and $V=\cot (\theta) / 2 a r$. Now (11), (12), (13), (32)-(35) and (36)-(37) produce non-zero components of the energy-momentum tensor:

$$
\begin{gathered}
T_{0}^{0}=m S+F(K), \\
T_{1}^{1}=F(K)-2 K F_{K}, \\
T_{2}^{2}=F(K)-2 K F_{K}, \\
T_{3}^{3}=F(K)-2 K F_{K}, \\
T_{1}^{0}=\frac{\cot (\theta)}{4 r} A^{3}, \\
T_{2}^{0}=-\frac{3}{4} A^{3}, \\
T_{3}^{0}=\frac{3}{4} \sin (\theta) A^{2}-\frac{1}{2} \cos (\theta) A^{1}, \\
T_{3}^{1}=-\frac{\cos (\theta)}{4 a} A^{0} .
\end{gathered}
$$

From (15), (16) and $T_{0}^{0}-T_{3}^{1}$ we get the complete system of Einstein equations. For diagonal elements we have:

$$
3 \frac{\dot{a}^{2}}{a^{2}}=8 \pi G[m S+F(K)]
$$




$$
2 \frac{\ddot{a}}{a}+\frac{\dot{a}^{2}}{a^{2}}=8 \pi G\left[F(K)-2 K F_{K}\right] .
$$

Expressions for non-diagonal elements:

$$
\begin{gathered}
\frac{\cot (\theta)}{4 r} A^{3}=0, \\
-\frac{3}{4} A^{3}=0, \\
\frac{3}{4} \sin (\theta) A^{2}-\frac{1}{2} \cos (\theta) A^{1}=0, \\
-\frac{\cos (\theta)}{4 a} A^{0}=0,
\end{gathered}
$$

where $A^{\mu}=\bar{\psi} \gamma^{5} \gamma^{\mu} \psi$ - components of the 4-pseudovector. This shows that the equations are identical for diagonal components, but non-diagonal components impose some additional conditions on either metric functions or spinor functions. These restrictions will be discussed in more detail in the next section. But this requires equations for spinor invariants. They are obtained from (36)-(37) and look like this:

$$
\begin{gathered}
\dot{S}+3 \frac{\dot{a}}{a} S+2 \Upsilon A^{0}=0, \quad \dot{P}+3 \frac{\dot{a}}{a} P-2[m+D] A^{0}=0, \\
\dot{A}^{1}+3 \frac{\dot{a}}{a} A^{1}+2 U A^{0}=0, \quad \dot{A}^{2}+3 \frac{\dot{a}}{a} A^{2}+2 V A^{0}=0 \\
\dot{A}^{0}+3 \frac{\dot{a}}{a} A^{0}+2 U A^{1}+2 V A^{2}+2[m+D] P-2 \Upsilon S=0 .
\end{gathered}
$$

In this instance $\left(\gamma^{5}\right)^{2}=1$. The first integral of this system is equal to:

$$
S^{2}+P^{2}+\left(A^{0}\right)^{2}-\left(A^{1}\right)^{2}-\left(A^{2}\right)^{2}=\frac{C}{a^{6}}, \quad C=\text { const. }
$$

\section{Restrictions on the spinor functions}

As mentioned earlier, the equations (48), (49), (50) and (51) impose restrictions on either the metric function or the spinor functions that are the solution of the equations (36)-(37). It follows from (48)-(51) that $A^{3}=0$ and $A^{0}=0$. A restriction on $A^{1}$ and $A^{2}$ looks like this:

$$
A^{1}=\frac{3}{2} \tan (\theta) A^{2} \text {. }
$$

Using (56) and equations from (48) to (51) we get the following:

$$
S^{2}+P^{2}+\left(A^{0}\right)^{2}-\left(A^{2}\right)^{2}\left[\frac{9}{4} \tan ^{2}(\theta)+1\right]=\frac{C}{a^{6}} .
$$


It can also be shown [24] that if $m \neq 0$, then:

$$
K=I=S^{2}=\frac{C_{1}}{a^{6}}, \quad C_{1}=\text { const }
$$

If $m=0$, then:

$$
K=\frac{C_{2}}{a^{6}}, \quad C_{2}=\text { const }
$$

for $K=I, K=J, K=I+J$ and $K=I-J$.

\section{Discussion}

Though there is a number of papers dealing with FLRW cosmological model with spinor fields, we did it again. Main idea was to see how the coordinate transformation effects the behavior of spacetime evolution and the spinor field. Moreover, mathematically it may help us to model different type of stars using the spinor field as a source field. In that sense this study is the beginning of the further studies that we plan to carry out in future.

\section{Conclusions}

Within the scope of spherically symmetric FLRW model we study the role of the spinor field in the evolution of the Universe. It is found that the usual transition from Cartesian coordinates to spherical ones leads to the appearance of non-zero non-diagonal components of the energy-momentum tensor. The presence of these components leads to some restrictions on spinor functions. However, these limitations may not always directly affect the solution of Einstein's equations. For example, if $K=I=S^{2}$, these restrictions will not affect the solution of the equations (25) and (26).

\section{Acknowledgments}

The publication has been prepared with the support of the "RUDN University Program 5-100" and funded by RFBR according to the research projects No. 18-07-00692 and No. 16-07-00766.

\section{References}

[1] B. Saha and G. N. Shikin, "Interacting Spinor and Scalar Fields in Bianchi Type $I$ Universe Filled with Perfect Fluid: Exact Self-consistent Solutions," General Relativity and Gravitation, vol. 29, pp. 1099-1112, 1997. DOI: $10.1023 / \mathrm{a}: 1018887024268$.

[2] B. Saha and G. N. Shikin, "Nonlinear Spinor Field in Bianchi type- $I$ Universe filled with Perfect Fluid: Exact Self-consistent Solutions," Journal of Mathematical Physics, vol. 38, pp. 5305-5318, 1997. DOI: $10.1063 / 1.531944$. 
[3] B. Saha, "Spinor field in Bianchi type- $I$ Universe: regular solutions," Physical Review D, vol. 64, p. 123 501, 2001. DOI: 10.1103/physrevd. 64.123501.

[4] B. Saha, "Nonlinear Spinor Field in cosmology," Physical Review D, vol. 69, p. 124006, 2004. DOI: 10.1103/physrevd.69.124006.

[5] B. Saha and T. Boyadjiev, "Bianchi type-I cosmology with scalar and spinor fields," Physical Review D, vol. 69, p. 124010, 2004. DOI: 10 . 1103/physrevd.69.124010.

[6] B. Saha, "Spinor fields in Bianchi type- $I$ Universe," Physics of Particlesand Nuclei., vol. 37, S13-S44, 2006. DOI: 10 . 1134 / s1063779606070021.

[7] N. J. Popławski, "Nonsingular, big-bounce cosmologyfrom spinor-torsion coupling," Physical Review D, vol. 85, p. 107502 , 2012. DOI: 10.1103/ physrevd.85.107502.

[8] M. O. Ribas, F. P. Devecchi, and G. M. Kremer, "Fermions as sources of accelerated regimes in cosmology," Physical Review D, vol. 72, p. 123502 , 2005. DOI: $10.1103 /$ physrevd.72.123502.

[9] B. Saha, "Nonlinear spinor field in Bianchi type- $I$ cosmology: inflation,isotropization, and late time acceleration," Physical Review D, vol. 74, p. 124030, 2006. DOI: 10.1103/physrevd.74.124030.

[10] B. Saha, "Spinor field and accelerated regimes in cosmology," Gravitation \& Cosmology, vol. 12, no. 46-47, pp. 215-218, 2006.

[11] B. Saha, "Nonlinear spinor field in Bianchi type- $I$ cosmology: accelerated regimes," Romanian Reports in Physics, vol. 59, pp. 649-660, 2007. arXiv: gr-qc/0608047.

[12] B. Saha, "Early inflation, isotropization and late-time acceleration of a Bianchi type-I universe," Physics of Particles and Nuclei, vol. 40, pp. 656-673, 2009. DOI: 10.1134/s1063779609050037.

[13] N. J. Popławski, "Big bounce from spin and torsion," General Releativity and Gravitation, vol. 44, p. 1007, 2012. DOI: 10.1007/s10714-0111323-2.

[14] N. J. Popławski, "Nonsingular Dirac particles in spacetime with torsion," Physics Letters B, vol. 690 , pp. 73-77, 2010. DOI: $10.1016 / \mathrm{j}$. physletb. 2010.04 .073$.

[15] L. Fabbri, "A Discussion on Dirac Field Theory, No-Go Theorems and Renormalizability," International Journal of Theoretical Physics, vol. 52, pp. 634-643, 2013. DOI: 10.1007/s10773-012-1370-9.

[16] L. Fabbri, "Conformal gravity with the most general ELKO matter," Physical Review D., vol. 85, p. 047 502, 2012. DOI: 10.1103/physrevd. 85.047502.

[17] S. Vignolo, L. Fabbri, and R. Cianci, "Dirac spinors in Bianchi-I $f(R)$ cosmology with torsion," Journal of Mathematical Physics, vol. 52, p. 112502 , 2011. DOI: 10.1063/1.3658865. 
[18] B. Saha, "Nonlinear Spinor Fields in Bianchi type- $I$ spacetime: Problems and Possibilities," Astrophysics and Space Science, vol. 357, p. 28, 2015. DOI: $10.1007 / \mathrm{s} 10509-015-2291-\mathrm{x}$.

[19] B. Saha, "Spinor field nonlinearity and space-time geometry," Physics of Particles and Nuclei, vol. 49, no. 2, pp. 146-212, 2018. DOI: 10.1134/ s1063779618020065.

[20] B. Saha, "Non-minimally coupled nonlinear spinor field in Bianchi type-I cosmology," European Physical Journal - Plus, vol. 134, p. 419, 2019. DOI: $10.1140 /$ epjp/i2019-12859-7.

[21] R. Cianci, L. Fabbri, and S. Vignolo, "Exact solutions for Weyl fermions with gravity," European Physical Journal - Plus, vol. 75, p. 478, 2015. DOI: $10.1140 / \mathrm{epjc} / \mathrm{s} 10052-015-3698-9$.

[22] K. A. Bronnikov, Y. P. Rybakov, and B. Saha, "Spinor fields in spherical symmetry. Einstein-Dirac and other space-time," European Physical Journal-Plus, vol. 135, p. 124, 2020. DOI: 10.1140/epjp/s13360-02000150-z.

[23] B. Saha, "Spinor fields in spherically symmetric space-time," European Physical Journal - Plus, vol. 133, p. 416, 2018. DOI: 10.1140/epjp/ i2018-12273-9.

[24] B. Saha, "Spinor Field Nonlinearity and Space-Time Geometry," Physics of Particles and Nuclei, vol. 49, no. 2, pp. 146-212, 2018. DOI: 10.1134/ S1063779618020065.

\section{For citation:}

S. Bijan, E. I. Zakharov, V.S. Rikhvitsky, Spinor field in a spherically symmetric Friedmann Universe, Discrete and Continuous Models and Applied Computational Science 28 (2) (2020) 131-140. DOI: 10.22363/2658-46702020-28-2-131-140.

\section{Information about the authors:}

Saha, Bijan - Doctor of Physical and Mathematical Sciences, assistant professor of the Institute of Physical Research and Technologies of Peoples' Friendship University of Russia (RUDN University), leading researcher at the Laboratory of Information Technologies of The Joint Institute for Nuclear Research (e-mail: bijan64@mail.ru, phone: +7(962)9095155, ORCID: https://orcid.org/0000-0002-6741-5409, ResearcherID: E-6604-2018, Scopus Author ID: 7202946069)

Zakharov, Evgeniy I. - student of the Institute of Physical Research and Technologies of Peoples' Friendship University of Russia (RUDN University) (e-mail: zakharov.eugene1998@gmail.com, phone: +7(901)7062157, ORCID: https://orcid.org/0000-0003-2458-6109)

Rikhvitsky, Victor S. - Master of physical and mathematical Sciences, Leading programmer of the Laboratory of Information Technologies of the Joint Institute for Nuclear Research (JINR) (e-mail: rqvtsk@mail.ru, ORCID: https://orcid.org/0000-0001-6597-7443, Scopus Author ID: 57190934347) 
УДК 524.834

PACS 98.80.Cq,

DOI: $10.22363 / 2658-4670-2020-28-2-131-140$

\title{
Спинорное поле в сферически симметричной Вселенной Фридмана
}

\author{
Саха Биджан $^{1,2}$, Е. И. Захаров ${ }^{1}$, В. С. Рихвицкий ${ }^{2}$ \\ ${ }^{1}$ Институт физических исследований и технологий \\ Российский университет дружбы народов \\ ул. Миклухо-Маклая, д. 6, Москва, 117198, Россия \\ 2 Лаборатория информационных технологий \\ Объединённый институт ядерных исследований \\ ул. Жолио-Кюри, д. 6, Дубна, Московская область, 141980, Россия
}

В последние годы спинорное поле используется многими авторами для решения некоторых актуальных вопросов современной космологии. Мотив использования спинорного поля в качестве источника гравитационного поля заключается в том, что спинорное поле может не только описывать различные этапы эволюции Вселенной, но и моделировать различные типы вещества, такие как идеальная жидкость и темная энергия. Кроме того, спинорное поле очень чувствительно к гравитационному, и в зависимости от гравитационного поля спинорное поле может реагировать по-разному, изменяя тем самым геометрию пространствавремени. В настоящей работе дается краткое описание нелинейного спинорного поля в модели Фридмана-Леметра-Робертсона-Уолкера (FLRW). Результаты сравниваются в декартовых и сферических координатах. Показано, что при переходе от декартовых координат к сферическим тензор энергии-импульса имеет дополнительные ненулевые недиагональные компоненты, которые могут накладывать ограничения как на спинорные функции, так и на метрические.

Ключевые слова: спинорное поле, модель FLRW, декартовы координаты, сферические координаты 